\title{
NEW THEOREMS ON THE PARITY OF PARTITION FUNCTIONS
}

\author{
BRUCE C. BERNDT ${ }^{1}$, AE JA YEE ${ }^{2}$, AND ALEXANDRU ZAHARESCU
}

\begin{abstract}
Working in the ring $A$ of formal power series in one variable over the field of two elements, we have recently established lower bounds for both the number of even values and the number of odd values for a wide variety of partition functions. Here, we introduce two further ideas, differential equations in $A$ and finite perturbations, to prove theorems on the parity of a considerably larger class of partition functions.
\end{abstract}

\section{INTRODUCTION}

Let $p(n)$ denote the ordinary partition function, i.e., the number of ways a positive integer $n$ can be represented as a sum of positive integers. It is expected that $p(n)$ is even approximately half of the time, or, more precisely,

$$
\#\{n \leq N: p(n) \text { is even }\} \sim \frac{1}{2} N,
$$

as $N \rightarrow \infty$. Numerical computations of T. R. Parkin and D. Shanks [13] seem to indicate that indeed (1.1) holds. The parity of $p(n)$ has been investigated by a number of authors, including O. Kolberg [6], J. Fabrykowski and M. V. Subbarao [4], M. Newman [8], L. Mirsky [7], J.-L. Nicolas and A. Sárközy [10]. Greatly improving on previous results, Nicolas, I. Z. Ruzsa, and Sárközy [9] proved that

$$
\#\{n \leq N: p(n) \text { is even }\} \gg \sqrt{N}
$$

and, for each $\epsilon>0$,

$$
\#\{n \leq N: p(n) \text { is odd }\} \gg \sqrt{N} e^{-(\log 2+\epsilon) \frac{\log N}{\log \log N}} .
$$

In an appendix to their paper [9], J.-P. Serre proved that

$$
\lim _{N \rightarrow \infty} \frac{\#\{n \leq N: p(n) \text { is even }\}}{\sqrt{N}}=\infty .
$$

At present, this is the best result for even values of $p(n)$. The lower bound (1.3) has been improved by S. Ahlgren [1] who proved that

$$
\#\{n \leq N: p(n) \text { is odd }\} \gg \frac{\sqrt{N}}{\log N} .
$$

The lower bound (1.5) is currently the best known result for odd values of $p(n)$.

\footnotetext{
${ }^{1}$ Research partially supported by grant MDA904-02-1-0065 from the National Security Agency.

${ }^{2}$ Research partially supported by a grant from the Number Theory Foundation.

${ }^{3}$ Mathematics Subject Classification 2000: Primary, 11P76; Secondary, 11P83.
} 
Subbarao [14] conjectured that in every arithmetic progression $n \equiv r(\bmod t)$ there are infinitely many values of $n$ such that $p(n)$ is even and that there are infinitely many values of $n$ for which $p(n)$ is odd. The most significant quantitative work on the parity of $p(n)$ in arithmetic progressions has been accomplished by K. Ono [11], [12] and Ahlgren [1] via the theory of modular forms. More details can be found in our first paper [2] and, of course, in the aforementioned authors' papers.

In [2], the authors developed new methods, which are simple and apply to more general partition functions. In particular, explicit results were proved in [2] on the parity of several classes of partition functions, such as: the number $p(r, s ; n)$ of partitions of $n$ into parts congruent to $r, s$, or $r+s$ modulo $r+s$; the number $c_{3}(n)$ of partitions of $n$ into three colors; the number of partitions of $n$ into parts which are coprime to a given fixed integer $b$; and the number of partitions of $n$ into parts which are square-free and coprime to a fixed integer $b$. For the latter problem, see also [15].

In the present paper we use new ideas, which allow us to develop the theory in a more systematic way, and this leads in turn to vast generalizations of many of the results from [2].

As in [2], all our work is effected in the ring $A$ of formal power series in one variable over the field of two elements $\mathbf{Z} / 2 \mathbf{Z}$. A key ingredient in the present work is a certain differential equation in $A$, which is studied in Section 3 below. Other essential tools in our investigation are two maps, introduced in Section 4. In Section 5 we define the concept of a finite perturbation, which gives more flexibility to our method. We then prove some general results on the parity of partition functions. In Section 6 we show how the results from the previous sections can be extended to the more general context of partition functions with colors. Lastly, in Section 8 we prove a theorem on the parity of the number of partitions with all parts belonging to a given arithmetic progression.

\section{The Ring $A$}

Let $A:=\mathbf{F}_{2}[[X]]$ be the ring of formal power series in one variable $X$ over the field with two elements $\mathbf{F}_{2}=\mathbf{Z} / 2 \mathbf{Z}$, i.e.,

$$
A=\left\{f(X)=\sum_{n=0}^{\infty} a_{n} X^{n}: a_{n} \in \mathbf{F}_{2} \text { for all } n\right\} .
$$

The ring $A$ is an integral domain. It is also a local ring, with maximal ideal generated by $X$. An element $f(X)=\sum_{n=0}^{\infty} a_{n} X^{n} \in A$ is invertible if and only if $a_{0}=1$. Since 0 and 1 are the only elements of $\mathbf{F}_{2}$, we may write any element $f(X) \in A$ in the form

$$
f(X)=X^{n_{1}}+X^{n_{2}}+\cdots,
$$

where the sum may be finite or infinite and $0 \leq n_{1}<n_{2}<\cdots$. For any $f(X) \in A$,

$$
f^{2}(X)=f\left(X^{2}\right)
$$

In other words, if $f(X)$ is given by $(2.2)$, then

$$
f^{2}(X)=X^{2 n_{1}}+X^{2 n_{2}}+\cdots
$$


We need to know the shape of a series $f(X)$, given by (2.2), when divided by $1-X$. For any integers $0 \leq a<b$, we have in $A$

$$
\frac{X^{a}+X^{b}}{1-X}=\frac{X^{a}\left(1-X^{b-a}\right)}{1-X}=X^{a}+X^{a+1}+\cdots+X^{b-1} .
$$

We put together pairs of consecutive terms $X^{n_{2 k+1}}+X^{n_{2 k+2}}$ and obtain

$$
\begin{gathered}
\frac{f(X)}{1-X}=\frac{X^{n_{1}}+X^{n_{2}}}{1-X}+\frac{X^{n_{3}}+X^{n_{4}}}{1-X}+\cdots+\frac{X^{n_{2 k+1}}+X^{n_{2 k+2}}}{1-X}+\cdots \\
=\left(X^{n_{1}}+X^{n_{1}+1}+\cdots+X^{n_{2}-1}\right)+\left(X^{n_{3}}+\cdots+X^{n_{4}-1}\right)+\cdots \\
+\left(X^{n_{2 k+1}}+\cdots+X^{n_{2 k+2}-1}\right)+\cdots
\end{gathered}
$$

If the sum from the right side of $(2.2)$ which defines $f(X)$ is finite, say $f(X)=X^{n_{1}}+$ $X^{n_{2}}+\cdots+X^{n_{s}}$, then

$$
\frac{f(X)}{1-X}=\left(X^{n_{1}}+X^{n_{1}+1}+\cdots+X^{n_{2}-1}\right)+\cdots+\left(X^{n_{s-1}}+X^{n_{s-1}+1}+\cdots+X^{n_{s}-1}\right),
$$

if $s$ is even, and

$$
\frac{f(X)}{1-X}=\left(X^{n_{1}}+\cdots+X^{n_{2}-1}\right)+\cdots+\left(X^{n_{s-2}}+\cdots+X^{n_{s-1}-1}\right)+\sum_{n=n_{s}}^{\infty} X^{n},
$$

if $s$ is odd. On $A$ we have a natural derivation which sends an $f(X) \in A$ to $f^{\prime}(X)=$ $\frac{d f}{d X} \in A$, namely,

$$
f(X)=\sum_{n=0}^{\infty} a_{n} X^{n} \rightarrow f^{\prime}(X)=\sum_{n=1}^{\infty} n a_{n} X^{n-1} .
$$

Note that for any $f(X) \in A$,

$$
f^{\prime \prime}(X)=0 \text {. }
$$

Let us also remark that for any $f(X)$ given in the form $(2.2)$, the condition

$$
f^{\prime}(X)=0
$$

is equivalent to the condition that all the exponents $n_{j}$ are even numbers.

\section{Solving a Differential Equation in $A$}

Relevant to our study is the differential equation

$$
Y^{\prime}(X)=h(X) Y(X)
$$

where $h(X)$ is a given nonzero element of $A$. There are two primary problems. The first one is to establish necessary and sufficient conditions in order for the equation (3.1) to have nonzero solutions $Y(X) \in A$. The second problem is to find all the nonzero solutions of (3.1), provided such solutions exist. The first problem is solved by the following observation. If $0 \neq Y(X) \in A$ is a solution to (3.1), then using (3.1) twice in combination with (2.10), we see that

$$
\begin{aligned}
0=Y^{\prime \prime}(X)=h^{\prime}(X) Y(X) & +h(X) Y^{\prime}(X)=h^{\prime}(X) Y(X)+h^{2}(X) Y(X) \\
= & \left(h^{\prime}(X)+h^{2}(X)\right) Y(X) .
\end{aligned}
$$


Since $A$ is an integral domain and $Y(X) \neq 0$, it follows that $h^{\prime}(X)+h^{2}(X)=0$, which can also be written as

$$
h^{\prime}(X)=h^{2}(X) .
$$

Note that (3.3) has the same shape as (3.1), with $Y(X)=h(X)$. Therefore if the equation (3.1) has nonzero solutions, then $h(X)$ itself is a solution of (3.1). This solves the first problem mentioned above: the equation (3.1) has nonzero solutions if and only if $h(X)$ satisfies (3.3), and in that case a particular solution to (3.1) is given by $Y(X)=h(X)$. We now proceed to find the general solution to (3.1), assuming that (3.3) holds. Note that the first nonzero term in $h(X)$ has an even exponent. Indeed, if this exponent is odd, say

$$
h(X)=X^{2 k+1}+X^{n_{1}}+X^{n_{2}}+\cdots,
$$

with $2 k+1<n_{1}<n_{2}<\cdots$, then the first nonzero term in $h^{\prime}(X)$ will be $X^{2 k}$ while the first nonzero term in $h^{2}(X)$ will be $X^{4 k+2}$, and $4 k+2>2 k$ for any $k \geq 0$. Therefore the first term in $h(X)$ has an even exponent, and we write $h(X)$ in the form

$$
h(X)=X^{2 k} h_{0}(X),
$$

for some integer $k \geq 0$ and $h_{0}(X)$ invertible in $A$. Next, we claim that $h_{0}$ is also a solution of (3.1). For, by (3.3) and (3.5),

$$
X^{2 k} h(X) h_{0}(X)=h^{2}(X)=h^{\prime}(X)=\left(X^{2 k}\right)^{\prime} h_{0}(X)+X^{2 k} h_{0}^{\prime}(X)=X^{2 k} h_{0}^{\prime}(X),
$$

which implies that

$$
h_{0}^{\prime}(X)=h(X) h_{0}(X),
$$

and so $h_{0}(X)$ is also a solution to (3.1). We note in passing that if the equation (3.1) has nonzero solutions, then it has a solution, namely $h_{0}(X)$, which is invertible in $A$.

Let us take now a general solution $Y(X)$ of (3.1). Since $h_{0}(X)$ is invertible in $A$, we may write $Y(X)$ in the form

$$
Y(X)=h_{0}(X) E(X)
$$

for some $E(X) \in A$. Say,

$$
E(X)=X^{b_{1}}+X^{b_{2}}+\cdots,
$$

with $0 \leq b_{1}<b_{2}<\cdots$, where, as usual, in such a representation the sum which appears on the right side of (3.9) may be finite or infinite. Differentiating (3.8), we obtain

$$
Y^{\prime}(X)=h_{0}^{\prime}(X) E(X)+h_{0}(X) E^{\prime}(X) .
$$

Using (3.1) and (3.7) in (3.10), we find that

$$
h(X) Y(X)=Y^{\prime}(X)=h(X) h_{0}(X) E(X)+h_{0}(X) E^{\prime}(X) .
$$

Using (3.8) in (3.11), we find that $h_{0}(X) E^{\prime}(X)=0$. This further implies $E^{\prime}(X)=0$, from which it follows that all the exponents $b_{j}$ which appear on the right side of $(3.9)$ are even.

Conversely, let $E(X)$ be given by (3.9) with all the $b_{j}$ even, and define $Y(X)$ by (3.8). Differentiating the equality (3.8), we obtain the relation (3.10). Here the last term vanishes by our assumption on $E(X)$, and so

$$
Y^{\prime}(X)=h_{0}^{\prime}(X) E(X) \text {. }
$$


Using (3.7) and (3.8) in (3.12), we see that

$$
Y^{\prime}(X)=h(X) h_{0}(X) E(X)=h(X) Y(X),
$$

and so $Y(X)$ satisfies (3.1). In conclusion, the general solution to (3.1) is given by (3.8), where $h_{0}(X)$ is uniquely determined in terms of $h(X)$ by $(3.5)$, and $E(X)$ is any element of $A$ with all the exponents $b_{j}$ in the representation (3.9) even numbers.

In applications to parity problems for partition functions, the differential equation (3.1) appears in a slightly different form. Namely, we will be interested in a function $F(X) \in A$ which is invertible in $A$ and satisfies an equality of the form

$$
X F^{\prime}(X)=F(X) H(X) \text {. }
$$

Here we suppose that $H(X)$ is known, and we want to be able to say something about $F(X)$. First, since $X$ divides the left side of (3.14), $X$ has to divide $H(X)$, since $F(X)$ is invertible. So we assume this condition in what follows. Then $H(X) / X$ will be a well defined element of $A$, denoted by $h(X)$, i.e.,

$$
H(X)=X h(X)
$$

The equation (3.14) is then equivalent to

$$
F^{\prime}(X)=h(X) F(X) .
$$

As we know, this equation has nonzero solutions if and only if $h(X)$ satisfies the condition (3.3). Let us write this condition in terms of our function $H(X)$. Differentiating (3.15) and using (3.3), we find that

$$
H^{\prime}(X)=h(X)+X h^{\prime}(X)=h(X)+X h^{2}(X) .
$$

Here we multiply the equality by $X$ and use (3.15) to find that

$$
X H^{\prime}(X)=H(X)+H^{2}(X) .
$$

Conversely, if $X$ divides $H(X)$, and $H(X)$ satisfies (3.18), then if $h(X)$ is defined by (3.15), the series $h(X)$ will satisfy (3.3). Indeed, from (3.15) it follows on one hand that

$$
H(X)+H^{2}(X)=X\left(h(X)+X h^{2}(X)\right),
$$

and on the other hand it follows that

$$
X H^{\prime}(X)=X\left(h(X)+X h^{\prime}(X)\right) .
$$

Combining (3.19), (3.20), and (3.18), we see that $h(X)$ satisfies (3.3). In conclusion the equation (3.14) has solutions with $F(X)$ invertible in $A$ if and only if $X$ divides $H(X)$ and $H(X)$ satisfies (3.18).

Assuming that these conditions are satisfied, we attempt to find the general solution of (3.14). We know that the first nonzero term in the series which gives $H(X)$ has an odd exponent, and so we may write

$$
H(X)=X^{2 k+1} H_{0}(X),
$$

where $k \geq 0$ is an integer, and $H_{0}(X)$ is an invertible element of $A$. Of course, by (3.15) it is clear that in the representations (3.5) and (3.21), $k$ is the same and $H_{0}(X)=h_{0}(X)$. We know that the general solution to (3.1) is given by (3.8), where $E(X)$, in the representation (3.9), has all its exponents $b_{j}$ even. Since the equations (3.1) and (3.16) 
are the same, except for the constraint on $F(X)$ to be invertible in $A$, we conclude that the general solution of (3.16), and therefore also the general solution of (3.14), is given by

$$
F(X)=H_{0}(X) E(X),
$$

where $E(X)$ is any invertible element of $A$ which, in the representation (3.9), has all its exponents $b_{j}$ even. We collect these results in the following theorem.

Theorem 3.1. Suppose that $H(X) \in A$ has a divisor $X$. The equation

$$
X F^{\prime}(X)=F(X) H(X)
$$

has solutions, with $F(X)$ invertible in $A$, if and only if $H(X)$ satisfies the condition

$$
X H^{\prime}(X)=H(X)+H^{2}(X) .
$$

If this condition holds, then the general solution $F(X)$ of (3.23), with $F(X)$ invertible in $A$, is given by

$$
F(X)=H_{0}(X) E(X)
$$

where $H_{0}(X)$ is the unique invertible element of $A$ for which

$$
H(X)=X^{2 k+1} H_{0}(X)
$$

for some integer $k \geq 0$, and $E(X)$ is any element of $A$ of the form

$$
E(X)=1+X^{b_{1}}+X^{b_{2}}+\cdots+X^{b_{j}}+\cdots,
$$

where $b_{j}$ is an even positive integer and $b_{j}<b_{j+1}$ for $j \geq 1$.

$$
\text { 4. The MAPS } S \mapsto F_{S}(X) \text { AND } S_{\text {odd }} \mapsto H_{S}(X)
$$

Let $\mathbf{N}=\{0,1,2,3, \ldots\}$ and $\mathbf{N}^{*}=\{1,2,3, \ldots\}$. For any subset $S$ of $\mathbf{N}^{*}$ we denote by $p_{S}(N)$ the number of partitions of $n$ with all parts in $S$. We begin with the generating function

$$
F_{S}(q):=1+\sum_{n=1}^{\infty} p_{S}(n) q^{n}=\prod_{n \in S} \frac{1}{1-q^{n}}
$$

and compute

$$
\log F_{S}(q)=-\sum_{n \in S} \log \left(1-q^{n}\right)=\sum_{n \in S} \sum_{m=1}^{\infty} \frac{q^{n m}}{m} .
$$

By applying the operator $q \frac{d}{d q}$ we obtain

$$
\frac{q F_{S}^{\prime}(q)}{F_{S}(q)}=q \frac{d}{d q}\left(\log F_{S}(q)\right)=\sum_{n \in S} \sum_{m=1}^{\infty} n q^{n m}=\sum_{k=1}^{\infty} q^{k} \sum_{n \in S, n \mid k} n=\sum_{k=1}^{\infty} \sigma_{S}(k) q^{k}
$$

where $\sigma_{S}(k)$ is the sum of those divisors of $k$ which belong to $S$. Let $F_{S}(X)$ be the image of $F_{S}(q)$ in $A$. Then, by $(4.1), F_{S}(X)$ will satisfy a differential equation of the form

$$
X F_{S}^{\prime}(X)=F_{S}(X) H_{S}(X),
$$

where $H_{S}(X)$ is the image in $A$ of the series on the far right side of (4.1). For any subset $S$ of $\mathbf{N}^{*}$ we denote by $S_{\text {odd }}$ the set of elements of $S$ which are odd numbers, and by $S_{\text {even }}$ the set of elements of $S$ which are even numbers. For any set $Y$, let $\mathcal{P}(Y)$ be the 
set of subsets of $Y$; we let $A^{\times}$denote the multiplicative group of invertible elements of $A$; and we denote by $\mathcal{H}$ the additive subgroup of $A$ consisting of those elements $H(X)$ which satisfy the equation $X H^{\prime}(X)=H(X)+H^{2}(X)$. We now describe a few properties of these maps.

(1) The map from $\mathcal{P}\left(\mathbf{N}^{*}\right)$ to $A^{\times}$given by $S \mapsto F_{S}(X)$ is bijective.

To prove that this map is injective, take two sets $S$ and $S^{\prime}$ such that $F_{S}(X)=F_{S^{\prime}}(X)$ and show, by induction on $n$, that $n$ belongs to $S^{\prime}$ if and only if it belongs to $S$. To show that the map is surjective, take any invertible element $F(X)=1+X^{n_{1}}+\cdots$ in $A$ and then construct a set $S$ for which $F_{S}(X)=F(X)$. In doing this, use again induction on $n$. Assume that the elements of $S$ which are strictly smaller than $n$ have been already chosen, so that the series $F_{S}(X)$ coincides with $F(X)$ modulo $X^{n}$. Then one decides whether to put $n$ in $S$ or not, so that $F_{S}(X)$ is congruent to $F(X)$ modulo $X^{n+1}$. In conclusion the map above is bijective. Also note that this map satisfies a multiplicative property. More precisely, if $S$ and $S^{\prime}$ are disjoint then $F_{S \cup S^{\prime}}(X)=F_{S}(X) F_{S^{\prime}}(X)$.

(2) We now turn to the map $S \mapsto H_{S}(X)$. For any $S$ and $S^{\prime}$ with $S_{\text {odd }}=S_{\text {odd }}^{\prime}$, we see that $\sigma_{S}(n)$ and $\sigma_{S^{\prime}}(n)$ have the same parity for any $n$. Therefore $H_{S}(X)=H_{S^{\prime}}(X)$. As a consequence, we have a well defined map, say $\Psi$, from $\mathcal{P}\left(\mathbf{N}_{\text {odd }}^{*}\right)$ to $\mathcal{H}$, such that $\Psi\left(S_{\text {odd }}\right)=H_{S}(X)$ for any subset $S$ of $\mathbf{N}^{*}$. Next, we show that the map $\Psi$ is bijective. To show that this map is injective, take two subsets $S$ and $S^{\prime}$ of $\mathbf{N}^{*}$ for which $H_{S}(X)=$ $H_{S^{\prime}}(X)$, that is, for which $\sigma_{S}(n)$ and $\sigma_{S^{\prime}}(n)$ have the same parity for each $n$. Then we prove, again by induction, that a positive odd integer $2 k+1$ belongs to $S$ if and only if it belongs to $S^{\prime}$. To show that the map $\Psi$ is surjective, let $H(X) \in \mathcal{H}$. From Theorem 3.1 we know that there exists $F(X)$ invertible in $A$ such that $X F^{\prime}(X)=F(X) H(X)$. By the bijection from (1) above we further deduce that there exists a subset $S$ of $\mathbf{N}^{*}$ for which $F_{S}(X)=F(X)$. Then $X F_{S}^{\prime}(X)=F_{S}(X) H(X)$, which in turn forces the equality $H(X)=H_{S}(X)$. So $\Psi$ is surjective. Note also that $\Psi$ satisfies an additive property. More precisely, if $S$ and $S^{\prime}$ are such that $S_{\text {odd }}$ and $S_{\text {odd }}^{\prime}$ are disjoint, then $\Psi\left(\left(S \cup S^{\prime}\right)_{\text {odd }}\right)=\Psi\left(S_{\text {odd }}\right)+\Psi\left(S_{\text {odd }}^{\prime}\right)$. In other words, $H_{S \cup S^{\prime}}(X)=H_{S}(X)+H_{S^{\prime}}(X)$.

(3) Using the results above one obtains generalizations of some of the results from [2]. For example, the proof given in Section 4 of [2], combined with the considerations from the present section, give the following results.

Corollary 4.1. For any set $S$ of positive integers which contains all the odd numbers, and for $N$ sufficiently large,

$$
\#\left\{n \leq N: p_{S}(n) \text { is even }\right\} \gg \sqrt{N} \text {. }
$$

Corollary 4.2. For any set $S$ of positive integers which contains all the odd numbers, we have, for each fixed $c>2 \log 2$ and all sufficiently large $N$,

$$
\#\left\{n \leq N: p_{S}(n) \text { is odd }\right\} \geq N^{\frac{1}{2}-\frac{c}{\log \log N}}
$$

In particular, the two results above apply to the number of partitions of a positive integer into odd parts, which equals the number of partitions of a positive integer into distinct parts. 
Corollary 4.3. There exists a set $S$ of positive integers, containing all the odd numbers, for which for $N$ sufficiently large,

$$
\#\left\{n \leq N: p_{S}(n) \text { is odd }\right\} \leq c \sqrt{N} .
$$

In order to prove Corollary 4.3, we appeal to Theorem 3.1 above which tells us that one particular solution to our differential equation is $H_{0}(X)$. Note that $\sigma_{S}(m)$ is odd if and only if each odd prime factor of $m$ has an even exponent in $m$. In other words, $\sigma_{S}(m)$ is odd if and only if $m=2^{r}(2 n+1)^{2}$ for some integers $r, n \geq 0$. It follows that

$$
H(X)=\sum_{n=1}^{\infty} X^{n^{2}}+\sum_{n=1}^{\infty} X^{2 n^{2}}
$$

and $H_{0}(X)=H(X) / X$. Next, by the bijection (1), there exists an $S$ for which $F_{S}(X)=$ $H_{0}(X)$. Of course, by the bijection (2) above it follows that this set $S$ contains all the odd numbers, since its associated series $H_{S}(X)$ coincides with the initial $H(X)$ given by (4.2). Finally, since $F_{S}(X)$ coincides with $H_{0}(X)$, which has less than $2 \sqrt{N}$ nonzero terms up to $X^{N}$, we conclude that there are less than $2 \sqrt{N}$ integers $n$ less than $N$ for which $p_{S}(n)$ is odd.

\section{Finite Perturbations}

For any two sets $S$ and $S^{\prime}$ we denote

$$
\Delta\left(S, S^{\prime}\right)=\left(S \backslash S^{\prime}\right) \cup\left(S^{\prime} \backslash S\right)=\left(S \cup S^{\prime}\right) \backslash\left(S \cap S^{\prime}\right) .
$$

We say that $S^{\prime}$ is a finite perturbation of $S$ provided $\Delta\left(S, S^{\prime}\right)$ is a finite set. If $\Delta\left(S, S^{\prime}\right)$ has an even number of elements, we say that $S^{\prime}$ is an even (finite) perturbation of $S$, while if the number above is odd, then we say that $S^{\prime}$ is an odd (finite) perturbation of $S$. In this section we take a set $S$ for which the sequence of terms from $H_{S}(X)$ is sparse. Then we consider sets $S^{\prime}$ for which $S_{\text {odd }}^{\prime}$ is a finite perturbation of $S_{\text {odd }}$, and investigate the parity of the corresponding partition functions $p_{S^{\prime}}(n)$. We emphasize that the corresponding series $H_{S^{\prime}}(X)$ are not sparse. In fact, it is impossible that, for two sets $S$ and $S^{\prime}$ with $\Delta\left(S_{\text {odd }}, S_{\text {odd }}^{\prime}\right)$ nonempty and finite, that the nonzero terms in both $H_{S}(X)$ and $H_{S^{\prime}}(X)$ have zero density. Indeed, let us assume that there are sets of positive integers $S$ and $S^{\prime}$ such that $S_{\text {odd }}^{\prime}$ is a finite perturbation of $S_{\text {odd }}, S_{\text {odd }}^{\prime} \neq S_{\text {odd }}$, and such that the nonzero terms in both series $H_{S}(X)$ and $H_{S^{\prime}}(X)$ have zero density. Say $\Delta\left(S_{\text {odd }}, S_{\text {odd }}^{\prime}\right)=\left\{b_{1}, b_{2}, \ldots, b_{l}\right\}$, where $1 \leq b_{1}<b_{2}<\cdots<b_{l}$. Choose any positive integer $a$ which is relatively prime to the product $b_{2} \cdots b_{l}$ and set $n=a b_{1}$. The point here is that $b_{1}$ is an odd divisor of $n$ which belongs to exactly one of the sets $S, S^{\prime}$, while any other odd divisor of $n$ either belongs to both sets $S, S^{\prime}$, or it belongs to none of them. Therefore $\sigma_{S}(n)$ and $\sigma_{S^{\prime}}(n)$ have different parity. It follows that for any such $n, X^{n}$ is a nonzero term in exactly one of the series $H_{S}(X)$ and $H_{S^{\prime}}(X)$, and hence it is a nonzero term in the sum $H_{S}(X)+H_{S^{\prime}}(X)$. Since the set of integers $a$ which are relatively prime to $b_{2} \cdots b_{l}$ has positive density, equal to $\varphi\left(b_{2} \cdots b_{l}\right) /\left(b_{2} \cdots b_{l}\right)$, we see that the set of nonzero terms in $H_{S}(X)+H_{S^{\prime}}(X)$ has positive density. Thus for at least one of the series $H_{S}(X)$ and $H_{S^{\prime}}(X)$, the sequence of nonzero terms has positive density. 
In the sequel, we then prove the following results, which generalize Theorems 5.1, 5.2 , and 5.3 from [2].

Theorem 5.1. Let $S$ be a set of positive integers. Then for each set of positive integers $S^{\prime}$ for which $S_{\text {odd }}^{\prime}$ is nonempty and is a finite perturbation of $S_{\text {odd }}$, there exists a constant $c_{1}>0$, depending on the set $\Delta\left(S, S^{\prime}\right)$ only, such that for large $N$,

$$
\#\left\{n \leq N: p_{S^{\prime}}(n) \text { is odd }\right\} \geq \frac{c_{1} B\left(H_{S}(X), N\right)}{D\left(H_{S}(X), N\right)} .
$$

Theorem 5.2. Let $S$ be a set of positive integers. Then for each set of positive integers $S^{\prime}$ for which $S_{\text {odd }}^{\prime}$ is an even finite perturbation of $S_{\text {odd }}$, there exist constants $c_{2}>0, c_{3}>$ 0 , depending on $\Delta\left(S, S^{\prime}\right)$ only, such that for $N$ sufficiently large,

$$
\#\left\{n \leq N: p_{S^{\prime}}(n) \text { is even }\right\} \geq \frac{\left.c_{2} N-c_{3} B\left(H_{S}(X), N\right)\right)}{1+B\left(H_{S}(X), N\right)} .
$$

Theorem 5.3. Let $S$ be a set of positive integers, containing at least one odd element, for which the sequence of nonzero terms of $H_{S}(X)$ has zero density. Then,

(i) for any set of positive integers $S^{\prime}$ for which $S_{\text {odd }}^{\prime}$ is nonempty and is a finite perturbation of $S_{\text {odd }}$, there are infinitely many positive integers $n$ for which $p_{S^{\prime}}(n)$ is odd, and

(ii) for any set of positive integers $S^{\prime}$ for which $S_{\text {odd }}^{\prime}$ is an even finite perturbation of $S_{\text {odd }}$, there are infinitely many positive integers $n$ for which $p_{S^{\prime}}(n)$ is even.

We can apply the results above to some of the sets $S$ considered in [2], for which the corresponding sequences of nonzero terms in $H_{S}(X)$ were sparse.

For example, we can derive in this way a result for the parity of the number of partitions of $n$ with all parts larger than 2002, say. Thus we take here $S^{\prime}=\{2002,2003, \ldots\}$, which is a finite perturbation of $S=\mathbf{N}^{*}$, and then use the estimates for $B\left(H_{S}(X), N\right)$ and $D\left(H_{S}(X), N\right)$ from [2]. Note that in this particular case $S_{\text {odd }}^{\prime}$ is an odd finite perturbation of $S_{\text {odd }}$; thus only Theorem 5.1 (and not Theorem 5.2) is applicable. If we consider more generally the number of partitions of $n$ with all parts larger than or equal to a given positive integer $r$, then this is realized by taking $S^{\prime}=\{r, r+1, \ldots\}$. Here again we let $S=\mathbf{N}^{*}$. Then Theorem 5.1 is always applicable, while Theorem 5.2 is applicable provided $r$ is congruent to 0 or 1 modulo 4 .

Similarly, we can prove a result on the parity of the number of partitions in square free parts, all larger than a given fixed integer $r$.

Before we begin the proofs of Theorems 5.1, 5.2, and 5.3, let us remark that, by the additive property of the map $\Psi$ from the previous section, it follows that for any sets $S$ and $S^{\prime}$,

and

$$
\begin{aligned}
& H_{S}(X)=H_{S \backslash S^{\prime}}(X)+H_{S \cap S^{\prime}}(X), \\
& H_{S^{\prime}}(X)=H_{S^{\prime} \backslash S}(X)+H_{S \cap S^{\prime}}(X),
\end{aligned}
$$

$$
H_{\Delta\left(S, S^{\prime}\right)}(X)=H_{S \backslash S^{\prime}}(X)+H_{S^{\prime} \backslash S}(X) .
$$

Keeping in mind that we work in $A$, we find from the equalities above that

$$
H_{S^{\prime}}(X)=H_{\Delta\left(S, S^{\prime}\right)}(X)+H_{S}(X) .
$$


Next, if $S_{\text {odd }}^{\prime}$ is a finite perturbation of $S_{\text {odd }}$, say, $\Delta\left(S_{\text {odd }}^{\prime}, S_{\text {odd }}\right)=\left\{b_{1}, b_{2}, \ldots, b_{l}\right\}$, then, by the same additive property of $\Psi$, we know that

$$
H_{\Delta\left(S, S^{\prime}\right)}(X)=H_{\Delta\left(S_{\text {odd }}, S_{\text {odd }}^{\prime}\right)}(X)=\sum_{j=1}^{l} H_{\left\{b_{j}\right\}}(X) .
$$

For each fixed $j$, we see that $\sigma_{\left\{b_{j}\right\}}(m)=1$, if $m$ is a multiple of $b_{j}$, and $\sigma_{\left\{b_{j}\right\}}(m)=0$, if $m$ is not a multiple of $b_{j}$. Thus,

$$
H_{\left\{b_{j}\right\}}(X)=\sum_{k=1}^{\infty} X^{k b_{j}}=\frac{X^{b_{j}}}{1-X^{b_{j}}} .
$$

If we introduce the rational function

$$
R_{S, S^{\prime}}(X)=\sum_{j=1}^{l} \frac{X^{b_{j}}}{1-X^{b_{j}}},
$$

then from (5.1)-(5.4), we conclude that

$$
H_{S^{\prime}}(X)=R_{S, S^{\prime}}(X)+H_{S}(X) .
$$

We now make a remark which will be needed later. Suppose an odd integer $b$ is chosen such that $b$ is divisible by each of the numbers $b_{1}, \ldots, b_{l}$. Then clearly

$$
\left(1-X^{b}\right) R_{S, S^{\prime}}(X)=: P_{S, S^{\prime}, b}(X)
$$

is a polynomial in $X$. We claim that, if $S_{\text {odd }}^{\prime}$ is an even finite perturbation of $S_{\text {odd }}$, in other words if $l$ is even, then $P_{S, S^{\prime}, b}(X) /(1-X)$ is also a polynomial in $X$. Indeed, for each $j$, the expression

$$
\frac{\left(1-X^{b}\right) X^{b_{j}}}{1-X^{b_{j}}}=\sum_{k=1}^{b / b_{j}} X^{k b_{j}}
$$

is a polynomial in $X$ which has an odd number of nonzero terms. If we add these polynomials for $j=1,2, \ldots, l$, then, $l$ being even, the total number of nonzero terms will be even. Some of these terms may cancel, but when they cancel they do so in pairs. We conclude that the polynomial $P_{S, S^{\prime}, b}(X)$ has an even number of nonzero terms. This implies that $P_{S, S^{\prime}, b}(X) /(1-X)$ is also a polynomial, as we saw in (2.7).

We are now ready for the proofs of the stated results. For the proof of Theorem 5.1, we use the known differential equation associated to $S^{\prime}$, namely,

$$
X F_{S^{\prime}}^{\prime}(X)=F_{S^{\prime}}(X) H_{S^{\prime}}(X) .
$$

Here we insert (5.5) for $H_{S^{\prime}}(X)$ to obtain

$$
X F_{S^{\prime}}^{\prime}(X)+F_{S^{\prime}}(X) R_{S, S^{\prime}}(X)=F_{S^{\prime}}(X) H_{S}(X) .
$$

Next, we choose an odd positive integer $b$ which is divisible by each of the numbers $b_{1}, \ldots, b_{l}$, and multiply both sides of $(5.7)$ by $1-X^{b}$. We find that, by (5.6),

$$
X\left(1-X^{b}\right) F_{S^{\prime}}^{\prime}(X)+F_{S^{\prime}}(X) P_{S, S^{\prime}, b}(X)=\left(1-X^{b}\right) F_{S^{\prime}}(X) H_{S}(X) .
$$

We now write $\left(1-X^{b}\right) F_{S^{\prime}}(X)$, which is an invertible element of $A$, in the form

$$
\left(1-X^{b}\right) F_{S^{\prime}}(X)=1+X^{n_{1}}+X^{n_{2}}+\cdots+X^{n_{j}}+\cdots
$$


We also write $H_{S}(X)$ as

$$
H_{S}(X)=X^{m_{1}}+X^{m_{2}}+\cdots+X^{m_{j}}+\cdots .
$$

Using the last two equalities in (5.8), we derive

$$
\begin{gathered}
X\left(1-X^{b}\right) F_{S^{\prime}}^{\prime}(X)+F_{S^{\prime}}(X) P_{S, S^{\prime}, b}(X)+\left(X^{n_{1}}+\cdots+X^{n_{k}}+\cdots\right) H_{S}(X) \\
=H_{S}(X)=X^{m_{1}}+X^{m_{2}}+\cdots+X^{m_{j}}+\cdots .
\end{gathered}
$$

The remainder of the proof is a counting argument, which goes as follows. If $F_{S^{\prime}}(X)$ is sparse, then both series $X\left(1-X^{b}\right) F_{S^{\prime}}^{\prime}(X)$ and $F_{S^{\prime}}(X) P_{S, S^{\prime}, b}(X)$ are sparse, the second one being sparse because $P_{S, S^{\prime}, b}(X)$ is a polynomial of fixed degree, as $N \rightarrow \infty$. Then the series $\left(X^{n_{1}}+\cdots+X^{n_{k}}+\cdots\right) H_{S}(X)$ on the left side of (5.9) cannot match the right side, given by $X^{m_{1}}+X^{m_{2}}+\cdots+X^{m_{j}}+\cdots$, if the $n_{j}$ are sparse. Thus, the $n_{j}^{\prime} s$ are not sparse. But then, the series $F_{S^{\prime}}(X)$ also cannot be sparse, because the series $1+X^{n_{1}}+X^{n_{2}}+\cdots+X^{n_{j}}+\cdots$ was obtained by multiplying $F_{S^{\prime}}(X)$ by $\left(1-X^{b}\right)$. A straightforward computation, based on the reasoning above, completes the proof of the theorem.

In order to prove Theorem 5.2, we start by introducing a series, call it $G_{S^{\prime}}(X)$, for which the set of nonzero terms is the complement of the set of nonzero terms in $F_{S^{\prime}}(X)$. Thus

$$
G_{S^{\prime}}(X)+F_{S^{\prime}}(X)=\frac{1}{1-X} .
$$

Since $p_{S^{\prime}}(n)$ is even if and only if $X^{n}$ appears as a nonzero term in $G_{S^{\prime}}(X)$, what we need in the following is to provide, for a given large positive integer $N$, a lower bound for the number of nonzero terms up to $X^{N}$ in $G_{S^{\prime}}(X)$. We differentiate (5.10) and obtain

$$
G_{S^{\prime}}^{\prime}(X)+F_{S^{\prime}}^{\prime}(X)=\frac{1}{(1-X)^{2}} .
$$

Then we solve for $G_{S^{\prime}}(X)$ and $G_{S^{\prime}}^{\prime}(X)$ in (5.10) and (5.11) and introduce the corresponding expressions in (5.7) to find that

$$
\frac{X}{(1-X)^{2}}-X G_{S^{\prime}}^{\prime}(X)=\left(\frac{1}{1-X}-G_{S^{\prime}}(X)\right)\left(R_{S, S^{\prime}}(X)+H_{S}(X)\right) .
$$

Next, with $b_{1}, \ldots, b_{l}$ having the same meaning as in the previous proof, we select an odd positive integer $b$ which is divisible by each of the numbers $b_{1}, \ldots, b_{l}$. We then multiply (5.12) by $\left(1-X^{b}\right)$, use (5.6), and rearrange terms in the form

$$
\begin{aligned}
X(1 & \left.-X^{b}\right) G_{S^{\prime}}^{\prime}(X)+\frac{P_{S, S^{\prime}, b}(X)}{1-X}+G_{S^{\prime}}(X) P_{S, S^{\prime}, b}(X)+\frac{\left(1-X^{b}\right) H_{S}(X)}{1-X} \\
& +G_{S^{\prime}}(X)\left(1-X^{b}\right) H_{S}(X)=\frac{X\left(1-X^{b}\right)}{(1-X)^{2}}=\frac{X+X^{2}+\cdots+X^{b}}{1-X} .
\end{aligned}
$$

Let $M$ be the number of nonzero terms up to $X^{N}$ in the series $G_{S^{\prime}}(X)$. The left side of (5.13) is a sum of five expressions. Let us denote, in order, by $M_{1}, \ldots, M_{5}$, the number of nonzero terms up to $X^{N}$ in these five series. Clearly, $G_{S^{\prime}}^{\prime}(X)$ has no more than $M$ nonzero terms up to $X^{N}$, and so $M_{1} \leq 2 M$. Recall that under our assumption that $S_{\text {odd }}^{\prime}$ is an even finite perturbation of $S_{o d d}, P_{S, S^{\prime}, b}(X) /(1-X)$ is a polynomial in $X$ of degree 
at most $b$, and so it follows that $M_{2} \leq b$. Next, again, since $P_{S, S^{\prime}, b}(X)$ is a polynomial of degree at most $b, M_{3} \leq(1+b) M$. The fourth term is a product of $H_{S}(X)$ and a polynomial of degree $b-1$; hence $M_{4} \leq b B\left(H_{S}(X), N\right)$. For the fifth term on the left side, we have $M_{5} \leq 2 M B\left(H_{S}(X), N\right)$. Now the point is that on the right side of (5.13), the numerator has an odd number of nonzero terms. As a consequence, its division by $(1-X)$ will have the shape described in (2.8). That is, for any $n \geq b, X^{n}$ appears as a nonzero term on the right side of our equality. So on the right side, the number of nonzero terms up to $X^{N}$ is at least $N-b$. Finally, by comparing the estimates obtained on the left side and, respectively, on the right side, we deduce Theorem 5.2.

We now turn to the proof of Theorem 5.3. Clearly, part (ii) follows directly from Theorem 5.2 above. In order to prove part (i), we use (5.7),

$$
X F_{S^{\prime}}^{\prime}(X)+F_{S^{\prime}}(X) R_{S, S^{\prime}}(X)=F_{S^{\prime}}(X) H_{S}(X) .
$$

Next, after choosing $b$ as before, we again obtain (5.8), i.e.,

$$
X\left(1-X^{b}\right) F_{S^{\prime}}^{\prime}(X)+F_{S^{\prime}}(X) P_{S, S^{\prime}, b}(X)=\left(1-X^{b}\right) F_{S^{\prime}}(X) H_{S}(X)
$$

Now the idea is is as follows. If we assume that $F_{S^{\prime}}(X)$ is a polynomial in $X$, then the entire left side of (5.14) will also be a polynomial in $X$. On the other hand, the product $\left(1-X^{b}\right) F_{S^{\prime}}(X)$ is also a polynomial, say,

$$
\left(1-X^{b}\right) F_{S^{\prime}}(X)=1+X^{n_{1}}+X^{n_{2}}+\cdots+X^{n_{k}}
$$

Since the sequence of nonzero terms of $H_{S}(X)$ is assumed to have zero density, there must be gaps larger than $n_{k}$ between consecutive terms in $H_{S}(X)$. Let us choose such a gap, say,

$$
H_{S}(X)=X^{m_{1}}+\cdots+X^{m_{s}}+X^{m_{s+1}}+\cdots
$$

where $m_{s+1}-m_{s}>n_{k}$. Consider now the product $\left(1-X^{b}\right) F_{S^{\prime}}(X) H_{S}(X)$. The point here is that the term $X^{n_{k}+m_{s}}$, obtained by multiplying $X^{n_{k}}$ from the right side of (5.15) with $X^{m_{s}}$ from the right side of (5.16), cannot be canceled by any other term. Therefore $X^{n_{k}+m_{s}}$ appears as one of the nonzero terms on the right side of (5.14). Clearly no term from the left side of (5.14) can have such a high exponent. In conclusion, $F_{S^{\prime}}(X)$ must have infinitely many nonzero terms, and this completes the proof of Theorem 5.3.

\section{Partition Functions with Colors}

In this section we consider partitions with colors. Given a map $C: \mathbf{N}^{*} \rightarrow \mathbf{N}$, we denote by $p_{C}(n)$ the number of partitions of $n$ with $C(m)$ colors, where $m$ is any positive integer. The generating for $p_{C}(n)$ is clearly

$$
F_{C}(q):=1+\sum_{n=1}^{\infty} p_{C}(n) q^{n}=\prod_{m=1}^{\infty} \frac{1}{\left(1-q^{m}\right)^{C(m)}} .
$$

As usual, $F_{C}(X)$ denotes the image of $F_{C}(q)$ in $A$. Denote by $\mathcal{C}$ the set of maps $C$ as above. Note that $\mathcal{C}$ has a natural structure of monoid with respect to addition, defined by $\left(C+C^{\prime}\right)(m)=C(m)+C^{\prime}(m)$ for any $m \in \mathbf{N}^{*}$. We remark that the map from $\mathcal{C}$ to $A^{\times}$given by $C \mapsto F_{C}(X)$ is a morphism of monoids, that is,

$$
F_{C+C^{\prime}}(X)=F_{C}(X) F_{C^{\prime}}(X),
$$


for any $C, C^{\prime} \in \mathcal{C}$. If we apply the operator $q \frac{d}{d q}$ to (6.1) and then send the resulting equality to $A$, we find, as before, that $F_{C}(X)$ satisfies a differential equation of the form

$$
X F_{C}^{\prime}(X)=F_{C}(X) H_{C}(X) .
$$

It is easy to see that the map from $\mathcal{C}$ to $\mathcal{H}$ given by $C \mapsto H_{C}(X)$ is also a morphism of monoids, in other words,

$$
H_{C+C^{\prime}}(X)=H_{C}(X)+H_{C^{\prime}}(X),
$$

for any $C, C^{\prime} \in \mathcal{C}$.

Note that $\mathcal{P}\left(\mathbf{N}^{*}\right)$ naturally injects in $\mathcal{C}$, by sending any subset $S$ of $\mathbf{N}^{*}$ to the map $C_{S}: \mathbf{N}^{*} \rightarrow \mathbf{N}$, given by $C_{S}(m)=1$ if $m \in S$ and $C_{S}(m)=0$ if $m \notin S$. In what follows we will identify each $S$ with the corresponding element $C_{S}$ of $\mathcal{C}$. Let us remark that $F_{S}(X)$ and $H_{S}(X)$ are left unchanged by this identification, in the sense that $F_{S}(X)=F_{C_{S}}(X)$ and $H_{S}(X)=H_{C_{S}}(X)$.

Next, we take any element $C$ of $\mathcal{C}$ and write it uniquely in the form

$$
C=2 C^{\prime}+S,
$$

where $C^{\prime} \in \mathcal{C}$ and $S$ is, via the above identification, a subset of $\mathbf{N}^{*}$. Here $C^{\prime}(m)=$ $\left[\frac{C(m)}{2}\right]$ for any $m \in \mathbf{N}^{*}$, while $S$ consists of those positive integers $m$ for which $C(m)$ is odd. In order to avoid possible confusion we sometimes denote by $S(C)$ the set $S$ defined by (6.5).

Combining (6.5) with (6.4) and using the fact that $A$ is a ring of characteristic 2 , we find that

$$
H_{C}(X)=H_{S(C)}(X),
$$

for any $C \in \mathcal{C}$. Therefore the series $F_{C}(X)$ and $F_{S(C)}(X)$, although distinct in general, are solutions of the same differential equation

$$
X Y^{\prime}(X)=Y(X) H_{S(C)}(X) .
$$

This fact has a number of consequences. More precisely, all our results for partition functions associated to sets $S$ of positive integers which have been obtained by studying the corresponding differential equations, can now be generalized to partition functions with colors.

For example, by applying these considerations to Corollaries 4.1 and 4.2 above, and using at the same time the perturbation results from Theorems 5.1 and 5.2, we derive the following results.

Theorem 6.1. Let $C: \mathbf{N}^{*} \rightarrow \mathbf{N}$ be such that $C(m)$ is odd for all but finitely many odd numbers $m$. Then, for each fixed $c$, with $c>2 \log 2$, and for any $N$ sufficiently large,

$$
\#\left\{n \leq N: p_{C}(n) \text { is odd }\right\} \geq N^{\frac{1}{2}-\frac{c}{\log \log N}} .
$$

Theorem 6.2. Let $C: \mathbf{N}^{*} \rightarrow \mathbf{N}$ be such that $C(m)$ is odd for all but an even number of odd numbers $m$. Then, for all $N$ large enough,

$$
\#\left\{n \leq N: p_{C}(n) \text { is even }\right\} \gg \sqrt{N} \text {. }
$$




\section{Partitions in Parts From a Given Arithmetic Progression}

In this section we investigate the parity of the number of partitions with all parts in a given arithmetic progression. Thus, given two positive integers $a, b$, we let $S$ be the arithmetic progression

$$
S:=\left\{n \in \mathbf{N}^{*}: n \equiv a(\bmod b)\right\},
$$

and consider the associated partition function $p_{S}$. Note that if $(a, b)=D>1$, then one has $p_{S}(n)=0$ unless $n$ is a multiple of $D$. On the other hand, if $n$ is a multiple of $D$, say, $n=D n_{1}$, and if we set

$$
S^{\prime}:=\left\{n \in \mathbf{N}^{*}: n \equiv a_{1}\left(\bmod b_{1}\right)\right\},
$$

where $a=D a_{1}, b=D b_{1}$, then $\left(a_{1}, b_{1}\right)=1$ and $p_{S}(n)=p_{S^{\prime}}\left(n_{1}\right)$. Thus the parity problem for $p_{S}$ reduces to the parity problem for $p_{S^{\prime}}$. For this reason, in what follows we may assume that $a$ and $b$ are relatively prime. Fix now $a$ and $b$ with $(a, b)=1$ and define $S$ by (7.1). We show that the nonzero terms of the corresponding series $H_{S}(X)$ have zero density. Then using Theorem 5.3 above we obtain the following result.

Theorem 7.1. Let $a, b$ be two relatively prime positive integers and let $S$ be the arithmetic progression given by (7.1). Then there are infinitely many positive integers $n$ for which $p_{S}(n)$ is even, and there are infinitely many positive integers $n$ for which $p_{S}(n)$ is odd.

In order to complete the proof of Theorem 7.1, it remains to prove the claim above on the density of nonzero terms of $H_{S}(X)$. Thus for any positive integer $m$ we examine the parity of $\sigma_{S}(m)$, with the intention of showing that $\sigma_{S}(m)$ is even for almost all values of $m$. By definition, we have

$$
\sigma_{S}(m)=\sum_{\substack{d \equiv a(\bmod b) \\ d \mid m}} d .
$$

We now bring into play Dirichlet characters $\chi$ modulo $b$, in order to put the right side of (7.3) in a more convenient form. By the orthogonality of characters, we know (see eq. (4), p. 31 of [3]) that

$$
\frac{1}{\phi(b)} \sum_{\chi} \overline{\bmod b}(a) \chi(n)= \begin{cases}1, & \text { if } n \equiv a(\bmod b) \\ 0, & \text { otherwise }\end{cases}
$$

Using (7.4) in (7.3), we deduce that

$$
\sigma_{S}(m)=\sum_{d \mid m} \frac{d}{\phi(b)} \sum_{\chi} \overline{\bmod b}(a) \chi(d) .
$$

Here we interchange the order of summation and put (7.5) in the form

$$
\phi(b) \sigma_{S}(m)=\sum_{\chi} \overline{\bmod b}(a) \sum_{d \mid m} d \chi(d) .
$$


Next, we use the fact that each character $\chi$ is multiplicative in order to write the corresponding inner sum on the right side of (7.6) as a product. If $m=p_{1}^{k_{1}} \cdots p_{s}^{k_{s}}$ is the prime decomposition of $m$, then we have

$$
\phi(b) \sigma_{S}(m)=\sum_{\chi} \overline{\bmod b}(a) G(\chi, m)
$$

where

$$
G(\chi, m)=\prod_{j=1}^{s}\left(1+p_{j} \chi\left(p_{j}\right)+\cdots+p_{j}^{k_{j}} \chi\left(p_{j}\right)^{k_{j}}\right) .
$$

Now let $K$ be the cyclotomic extension of the field $\mathbf{Q}$ of rational numbers, obtained by adjoining the roots of unity of order $\phi(b)$, and denote by $O_{K}$ the ring of integers of $K$. Then $\chi(n) \in O_{K}$ for any Dirichlet character $\chi \bmod b$ and any positive integer $n$. It follows that $G(\chi, m) \in O_{K}$ for any $\chi$ and $m$ as above. Fix a prime ideal $\mathcal{I}$ of $O_{K}$ which divides 2 , and denote by $e$ the ramification index of $\mathcal{I}$. Denote also by $k$ the exponent of 2 in $\phi(b)$, and then choose a positive integer $m$. If $\sigma_{S}(m)$ is odd, then the exact power of 2 which divides the left side of (7.7) is $2^{k}$, and consequently the exponent of $\mathcal{I}$ on the left side of (7.7) equals $k e$. We will show that for almost all $m$, the exponent of $\mathcal{I}$ on the right side of (7.7), call it $l_{m}$, is at least $k e+1$. This will force $\sigma_{S}(m)$ to be even for all these values of $m$. For any positive integer $m$ and any Dirichlet character $\chi \bmod b$, denote by $l_{\chi, m}$ the exponent of $\mathcal{I}$ in $G(\chi, m)$. Since the numbers $\bar{\chi}(a)$ are algebraic integers, it follows that for any $m$,

$$
l_{m} \geq \min _{\chi} l_{\chi, m}
$$

We will show that for any Dirichlet character $\chi \bmod b$,

$$
\lim _{M \rightarrow \infty} \frac{\#\left\{m \leq M: l_{\chi, m} \leq k e\right\}}{M}=0 .
$$

Then from (7.9) and (7.10) it will follow that

$$
\lim _{M \rightarrow \infty} \frac{\#\left\{m \leq M: l_{m} \leq k e\right\}}{M}=0
$$

and we are done. It remains to prove (7.10). In what follows, let $\chi$ be a fixed Dirichlet character mod $b$. We need to show that

$$
\#\left\{m \leq M: l_{\chi, m} \leq k e\right\} \leq \epsilon M,
$$

for any $\epsilon>0$ and any $M$ large enough in terms of $\epsilon$. Fix $\epsilon>0$. Denote by $\mathcal{D}_{\epsilon}$ the set of those positive integers $m$ which have a divisor $d \geq 1+2 / \epsilon$ for which $d^{2}$ is also a divisor of $m$. We claim that for any positive integer $M$,

$$
\#\left\{1 \leq m \leq M: m \in \mathcal{D}_{\epsilon}\right\} \leq \frac{\epsilon M}{2} .
$$


Indeed, for any positive integers $d$ and $M$, there are $\left[M / d^{2}\right]$ positive integers $m \leq M$ which are divisible by $d^{2}$. Therefore

$$
\#\left\{1 \leq m \leq M: m \in \mathcal{D}_{\epsilon}\right\} \leq \sum_{1+2 / \epsilon \leq d \leq \sqrt{M}}\left[\frac{M}{d^{2}}\right]<M \sum_{d \geq 1+2 / \epsilon} \frac{1}{d^{2}} \leq M \int_{2 / \epsilon}^{\infty} \frac{1}{X^{2}} d X=\frac{\epsilon M}{2}
$$

which proves (7.13).

We now make the following observation. Let $m$ be a positive integer and let $m=$ $p_{1}^{k_{1}} \cdots p_{s}^{k_{s}}$ be the prime decomposition of $m$. Suppose there is a $j \in\{1, \ldots, s\}$ such that $p_{j} \equiv 1(\bmod b), p_{j}$ is odd, and such that $k_{j}=1$. Then $\chi\left(p_{j}\right)=1$, and the corresponding factor $\left(1+p_{j} \chi\left(p_{j}\right)+\cdots+p_{j}^{k_{j}} \chi\left(p_{j}\right)^{k_{j}}\right)$ from the right side of (7.8) reduces to $1+p_{j}$, which is an even number, and so it is divisible by $\mathcal{J}^{e}$. Suppose now that there are at least $k+1$ distinct prime factors of $m$, namely, $p_{1}, \ldots, p_{k+1}$, such that for any $j \in\{1, \ldots, k+1\}$, we have $p_{j} \equiv 1(\bmod b), p_{j}$ is odd and $k_{j}=1$. Then, for each $j \in\{1, \ldots, k+1\}$, the corresponding factor on the right side of (7.8) is divisible by $\mathcal{J}^{e}$. The exponent of $\mathcal{J}$ in each of the remaining $s-k-1$ factors on the right side of $(7.8)$ is nonnegative, and so $l_{\chi, m} \geq(k+1) e$ for such values of $m$. Denote by $\mathcal{G}$ the set of positive integers $m$ having this property, that is, integers $m$ which have at least $k+1$ distinct odd prime factors $p_{j}$ which are congruent to 1 modulo $b$ and such that none of the numbers $p_{j}^{2}$ divides $m$. The discussion above shows that

$$
l_{\chi, m} \geq(k+1) e
$$

for any $m \in \mathcal{G}$.

Next, we fix a prime number $p_{0} \geq k+2$ such that $p_{0}$ does not divide $b$, and consider the arithmetic progressions modulo $b p_{0}$ given by

$$
S_{r}:=\left\{(1+r b)+b p_{0} n: n \in \mathbf{N}\right\},
$$

for $r=0,1, \ldots, p_{0}-1$. Note that the sets $S_{r}$ are disjoint. Since $b$ is not a multiple of $p_{0}$, there is exactly one value of $r$, call it $r_{0}$, for which $p_{0}$ divides $1+r_{0} b$. Since $p_{0} \geq k+2$, we may choose a subset $\mathcal{R}$ of $\left\{0,1, \ldots, p_{0}-1\right\}$, not containing $r_{0}$, and having $k+1$ elements. The set $\mathcal{R}$ will be fixed in what follows. Since $\left(1+r b, b p_{0}\right)=1$ for any $r \in \mathcal{R}$, by the Prime Number Theorem for arithmetic progressions (see, for example, [3], Ch. 20, p. 123) we know that as $x \rightarrow \infty$,

$$
\#\left\{p \leq x: p \text { prime }, p \in S_{r}\right\} \sim \frac{x}{\phi\left(b p_{0}\right) \log x} .
$$

For any $r \in \mathcal{R}$, denote by $\mathcal{E}(\epsilon, r)$ the set of positive integers which are not divisible by any prime number $p \geq 1+2 / \epsilon$ which belongs to $S_{r}$. Taking (7.17) into account, by sieve theory (see Halberstam and Richert's text [5], Thm. 8.4, p. 236) we know that each such set $\mathcal{E}(\epsilon, r)$ has zero density. Thus, there exists an $M_{0}$ depending on $a, b, p_{0}$, and $\epsilon$, such that

$$
\#\{m \leq M: m \in \mathcal{E}(\epsilon, r)\} \leq \frac{\epsilon M}{2(k+1)}
$$


uniformly for any $M \geq M_{0}$ and any $r \in \mathcal{R}$. Set

$$
\mathcal{H}=\mathcal{D}_{\epsilon} \bigcup_{r \in \mathcal{R}} \mathcal{E}(\epsilon, r)
$$

By (7.13) and (7.18), we see that

$$
\#\{m \leq M: m \in \mathcal{H}\} \leq \epsilon M,
$$

for any $M \geq M_{0}$. Finally, we claim that any positive integer $m$ which does not belong to $\mathcal{H}$, will necessarily lie in $\mathcal{G}$. Indeed, let $m \notin \mathcal{H}$. Then for each $r \in \mathcal{R}, m \notin \mathcal{E}(\epsilon, r)$; thus $m$ has a prime factor $q_{r} \geq 1+2 / \epsilon$ with $q_{r} \in S_{r}$. These prime factors $q_{r}$, with $r \in \mathcal{R}$ are distinct, and each of them is congruent to 1 modulo $b$. Moreover, since $m \notin \mathcal{D}_{\epsilon}$ and each $q_{r}$ satisfies the inequality $q_{r} \geq 1+2 / \epsilon$, it follows that none of the numbers $q_{r}^{2}$ divides $m$. In conclusion $m$ lies in $\mathcal{G}$, and so our claim is proved. Using this claim, in combination with (7.15) and (7.20), we deduce that (7.12) holds for any $M \geq M_{0}$. This completes the proof of the theorem.

\section{REFERENCES}

[1] S. Ahlgren, Distribution of parity of the partition function in arithmetic progressions, Indag. Math. (N.S.) 10 (1999), 173-181.

[2] B. C. Berndt, A. J. Yee, and A. Zaharescu, On the parity of partition functions, submitted for publication.

[3] H. Davenport, Multiplicative Number Theory, Third Edition, Springer, 2000.

[4] J. Fabrykowski and M. V. Subbarao, Some new identities involving the partition function $p(n)$, in Number Theory, R. A. Mollin, ed., Walter de Gruyter, New York, 1990, pp. 125-138.

[5] H. Halberstam and H. E. Richert, Sieve Methods, LMS Monographs No. 4, Academic Press, London, 1974.

[6] O. Kolberg, Note on the parity of the partition function, Math. Scand. 7 (1959), 377-378.

[7] L. Mirsky, The distribution of values of the partition function in residue classes, J. Math. Anal. Appl. 93 (1983), 593-598.

[8] M. Newman, Periodicity modulo $m$ and divisibility properties of the partition function, Trans. Amer. Math. Soc. 97 (1960), 225-236.

[9] J.-L. Nicolas, I. Z. Ruzsa and A. Sárközy, On the parity of additive representation functions. With an appendix by J-P. Serre, J. Number Theory 73 (1998), 292-317.

[10] J.-L. Nicolas and A. Sárközy, On the parity of partition functions, Illinois J. Math. 39 (1995), $586-597$.

[11] K. Ono, Parity of the partition function in arithmetic progressions, J. Reine Angew. Math. 472 (1996), 1-15.

[12] K. Ono, The partition function in arithmetic progressions, Math. Ann. 312 (1998), 251-260.

[13] T. R. Parkin and D. Shanks, On the distribution of parity in the partition function, Math. Comp. 21 (1967), 466-480.

[14] M. V. Subbarao, Some remarks on the partition function, Amer. Math. Monthly 73 (1966), $851-854$.

[15] A. Zaharescu, On the parity of the number of partitions in square free parts, Ramanujan J., to appear.

Department of Mathematics, University of Illinois, 1409 West Green Street, UrBANA, IL 61801, USA

E-mail address: berndt@math.uiuc.edu

E-mail address: yee@math.uiuc.edu

E-mail address: zaharesc@math.uiuc.edu 